\title{
Nonalcoholic steatohepatitis: An evolving diagnosis
}

\author{
Brent A Neuschwander-Tetri MD
}

\begin{abstract}
BA Neuschwander-Tetri. Nonalcoholic steatohepatitis: An evolving diagnosis. Can J Gastroenterol 2000;14(4):321-326. Nonalcoholic steatohepatitis (NASH) is a histological diagnosis applied to a constellation of liver biopsy findings that develop in the absence of alcohol abuse. Steatosis, a mixed cellular inflammatory infiltrate across the lobule, evidence of hepatocyte injury and fibrosis are the findings that can be seen. This entity is often identified during evaluation of elevated aminotransferases after exclusion of viral, metabolic and other causes of liver disease. Obesity is a major risk factor for NASH. The role of diabetes is less certain, although evidence is accumulating that hyperinsulinism may play an important pathophysiological role. Patients sometimes suffer from right upper quadrant abdominal pain and fatigue; examination may reveal centripetal obesity and hepatomegaly. Although patients are often discovered because of persistent aminotransferase elevations, these enzymes can be normal in NASH. When they are elevated, the alanine aminotransferase level is typically significantly greater than the aspartate aminotransferase level. This can be particularly helpful for excluding occult alcohol abuse. Imaging studies identify hepatic steatosis when the amount of fat in the liver is significant; however, imaging does not distinguish benign steatosis from NASH. Ultimately a liver biopsy is needed to diagnose NASH. The biopsy may be useful for establishing prognosis based on the presence or absence of fibrosis and for excluding other unexpected causes of liver enzyme elevations. Weight loss is the mainstay of treatment for obese patients. About $15 \%$ to $40 \%$ of NASH patients develop fibrosis; how many of these cases progress to cirrhosis is unknown, but about $1 \%$ of liver transplants are performed with a pretransplant diagnosis of $\mathrm{NASH}$.
\end{abstract}

Key Words: Liver transplantation; Nonalcoholic steatohepatitis; Steatosis

\section{La stéatose hépatique non alcoolique : un diagnostic en évolution}

RÉSUMÉ: La stéatose hépatique non alcoolique (SHNA) est un diagnostic histologique qui s'applique, à la suite d'une biopsie du foie, à de nombreux troubles qui se manifestent en l'absence d'abus d'alcool. On peut remarquer la présence de stéatose, d'un infiltrat inflammatoire cellulaire mixte à la grandeur du lobule, de lésions dans les hépatocytes et de fibrose. La maladie est souvent décelée au cours de l'investigation pour des taux élevés de transaminases après que des causes virales, métaboliques ou d'autre nature de maladie hépatique ont été exclues. L'obésité constitue un facteur de risque important de la SHNA. Le rôle du diabète est moins certain, mais de plus en plus de données semblent indiquer que l'hyperinsulinie pourrait jouer un rôle physiopathologique important. Les patients se plaignent parfois de douleurs abdominales dans le quadrant supérieur droit et de fatigue; l'examen peut révéler la présence d'obésité centripète et une hépatomégalie. Même s'il arrive souvent que la maladie est diagnostiquée à cause de taux élevés de transaminases, celles-ci peuvent se trouver en concentration normale dans la SHNA. Dans le cas contraire, le taux d'alanine-aminotransférase est généralement beaucoup plus élevé que celui de l'aspartate-aminotransférase. Il s'agit là d'une observation qui peut s'avérer fort utile pour exclure l'abus caché d'alcool. L'imagerie exploratoire parvient à déceler la stéatose hépatique en présence d'une quantité importante de graisses dans le foie mais ne permet pas de faire la distinction entre la stéatose bénigne et la SHNA. Finalement, il faut procéder à une biopsie du foie pour confirmer le diagnostic de la SHNA. L'examen peut permettre d'établir un pronostic fondé sur la présence ou l'absence de fibrose ou d'exclure des causes insoupçonnées de taux élevés d'enzymes hépatiques. La pierre angulaire du traitement chez les patients obèses est la perte de poids. La fibrose s'installe dans 15 à 40 \% des cas, mais on ne sait pas combien évoluent vers la cirrhose. Ce que l'on sait, c'est que $1 \%$ environ des transplantations du foie ont pour diagnostic préopératoire la SHNA.
$\mathrm{N}$ onalcoholic steatohepatitis (NASH), a common cause of liver enzyme abnormalities and occasionally progressive liver disease, is a diagnosis established by liver biopsy after the exclusion of other causes of liver disease. This re- view discusses who is at risk for NASH, why it develops, how it is diagnosed and what the physician can recommend to patients with NASH. Because the inciting factor or factors that cause NASH are unknown, the cumulative clinical knowl-

This mini-review was prepared from a presentation made at the American Congress of Gastroenterology, October 1998, Boston, Massachusetts Department of Internal Medicine, Saint Louis University School of Medicine, Saint Louis, Missouri, USA

Correspondence: Dr Brent A Neuschwander-Tetri, Division of Gastroenterology Hepatology, Saint Louis University,

3635 Vista Avenue, Saint Louis, Missouri 63110, USA. Telephone 314-577-8764, fax 314-577-8125, e-mail tetriba@slu.edu

Received for publication March 19, 1999. Accepted March 24, 1999 
edge is unavoidably descriptive. However, as specific causes of the underlying disorders are identified, NASH will likely be recognized as the liver's response to diverse metabolic defects, nutritional deficiencies or perhaps even unrecognized infections.

\section{RISK FACTORS FOR NASH}

The development of NASH is probably the result of a stress or injury to the liver in which there is pre-existing steatosis. To this extent, hepatic steatosis is a necessary precursor lesion for the inflammation, cellular injury and fibrosis that can occur in NASH. Body habitus is the primary predictor of benign hepatic steatosis; hepatic steatosis is found in $21 \%$ of lean healthy males and nearly all morbidly obese individuals. In the absence of morbid obesity but including those with a body mass more than $10 \%$ above lean body weight, about $75 \%$ have some degree of hepatic steatosis. In a curious analogy to the associated risk of coronary artery disease, the body fat distribution predicts the presence of hepatic steatosis. Body fat distributed about the hips and legs (pear shaped) is probably not a risk factor for steatosis, whereas a high ratio of abdominal fat to hip fat has been repeatedly found to be a significant risk factor for hepatic steatosis. The young are not protected; hepatic steatosis is found in obese children in a similar frequency to that of adults.

Several reports have described small groups of patients with NASH with the aim of identifying associated risk factors. Recognizing that these series are subject to referral bias and that only patients with elevated liver enzymes are typically evaluated, the risk factors for developing NASH have been found to be similar to the risk factors for benign steatosis. In earlier studies, patients with NASH were thought to be primarily obese, diabetic, female and hyperlipidemic. However, recent surveys found that not all patients have these 'classic' risk factors (1). Moreover, protocol liver biopsies obtained before surgical intervention for morbid obesity have shown significant NASH even in the absence of liver enzyme abnormalities (2).

These caveats aside, obesity is clearly the most significant risk factor for the development of NASH (3-6). The overall prevalence of NASH in obese patients has been estimated in both surgical series and autopsy studies and found to range from $8.7 \%$ to $18.5 \%(2,7)$. Careful exclusion of coexisting risk factors, such as alcohol use, diabetes, protein malnutrition and drug toxicity, has confirmed that obesity alone is a major risk factor for the development of NASH.

Type II diabetes is frequently associated with hepatic steatosis. Steatosis is found in about one-third of nonobese type II diabetics at autopsy; as a group, patients found to have hepatic steatosis by sonography are more likely to have glucose intolerance and elevated baseline insulin levels than patients without ultrasonographic evidence of hepatic steatosis $(7,8)$. The role of diabetes as a cause of NASH, liver fibrosis and ultimately cirrhosis in the absence of coexisting risk factors such as obesity continues to be debated. Arguments persist that diabetes alone is a risk factor for the development of cirrhosis, yet convincing evidence implicating diabetes in the development of chronic liver disease in the absence of other risk factors is still lacking $(9,10)$. Hepatic steatosis is relatively uncommon in type I diabetes, and when steatosis is present in type I diabetics, it correlates with consistently poor glycemic control.

Hyperlipidemia, a condition that broadly includes elevations of serum cholesterol, serum triglycerides or both, has been thought to be a risk factor or clinical marker for the development of NASH. Severe genetic disorders of serum lipid metabolism and the lipodystrophies are clearly associated with the development of NASH and the progression to end-stage liver disease (11). These rare diseases are characterized by abnormal mobilization of fat from peripheral stores, which greatly increases the flux of fatty acids through the liver. Whether the liver expresses abnormal fat metabolism in the lipodystrophies or is a passive target of the unusually high traffic of fatty acids is not known. However, in the patient lacking one of these unusual disorders, a direct association between hypertriglyceridemia and NASH lacks convincing epidemiological evidence. This may be largely due to the dominant and confounding effects of obesity and dietary habits. No study has shown an association between hypercholesterolemia and NASH.

Of the risk factors for NASH identified in early surveys, female sex was overemphasized and remains unconfirmed. In fact, the prevalence of NASH is equal among men and women at autopsy, and the prevalence of hepatic steatosis found by computed tomographic (CT) imaging is equal among men and women $(7,12)$. More recent surveys of patients with NASH have accordingly not shown an overwhelming predilection for the syndrome among women.

Several drugs have been implicated in the development of NASH. Most common are the corticosteroids and estrogens. Estrogens increase hepatic triglyceride synthesis, which may outpace the capacity of the liver to secrete triglyceride. What may seem contradictory is the association between tamoxifen use and the development of NASH. Well characterized cases have been described in women using tamoxifen as an estrogen antagonist as adjuvant therapy for breast cancer. However, tamoxifen may also exert weak estrogenic activity depending on the target tissue, and this activity may be responsible for its association with NASH. L-asparaginase may also be an important cause in a minority of patients.

\section{PATHOPHYSIOLOGY OF NASH}

If fat accumulation in the liver provides the necessary setting for the development of inflammation, injury and fibrosis, then an understanding of why fat accumulates in the liver may be important for identifying effective therapies. Fat accumulates in the liver when the delivery of fatty acids to the hepatocyte exceeds the capacity of the liver to metabolize them. There are two major mechanisms by which fatty acids are delivered to the liver: albumin-bound circulating fatty acids derived from peripheral adipose tissue, or synthesis within the liver in response to protein or carbohydrate excess. Similarly there are two major mechanisms of fatty acid 
disposal: mitochondrial beta-oxidation to form ATP and ketone bodies or secretion as triglyceride in very low density lipoprotein (VLDL). Derangements of these pathways, whether acquired, inherited or induced by diet and drugs, may cause fat to accumulate in the liver in the form of triglyceride. Fat can be envisioned as cycling from peripheral adipose tissue to the liver as fatty acids and delivery from the liver to peripheral sites as triglyceride. Dietary fat enters the circulation as triglyceride in chylomicrons and is not a direct source of fat in the liver.

The delivery of fat to the liver is considered first. Triglycerides are released from adipose tissue into the circulation as free fatty acids through the actions of lipoprotein lipases in a process controlled by adipocyte cAMP levels. Adrenaline, noradrenaline, adrenocorticotrophic hormone, glucagon, corticosteroids and methylxanthines (eg, caffeine) all stimulate adipocyte adenyl cyclase, which increases lipolysis via a hormone-sensitive lipase. In the fed state, insulin exerts an opposing effect by increasing phosphodiesterase activity, thereby depleting intracellular cAMP and inhibiting fatty acid release. Insulin is also required for the reincorporation of fatty acids into triglyceride in both the liver and peripheral stores. After feeding, fat release from peripheral stores is inhibited and fat release from the liver is promoted.

Free fatty acids released from peripheral stores are conveyed by albumin to tissues capable of fatty acid uptake and used as a metabolic fuel. The liver is the major site of uptake, but the heart and skeletal muscle are also highly dependent on fatty acids and remove a fraction from the circulation. The uptake of fatty acids by the liver is passive, and in the absence of feedback regulation, the liver cannot decrease fatty acid uptake, even when the capacity to catabolize fatty acids or re-esterify them and secrete them back into the circulation as VLDL is overwhelmed. Derangements in peripheral lipolysis thus directly influence the flux of fat through the liver. De novo synthesis of fatty acids within the liver is the other major source of fat. Conversion to fatty acids and then to triglyceride is thus the primary fate of most excess dietary carbohydrate and protein.

Although delivery of fatty acids to the liver and fatty acid synthesis in the liver are the major contributors to liver fat, not all liver triglyceride is derived from fatty acids. A minor fraction of liver triglyceride is derived from directly endocytosed lipoprotein remnants such as low density lipoprotein (LDL), intermediate density lipoprotein (IDL), VLDL remnants and chylomicron remnants. This pathway is probably not a major source of liver fat accumulation and the development of NASH except in the case of the rare lipodystrophies.

The fate of fat within the liver is considered next. Betaoxidation of fatty acids is one of the major fates of fatty acids in the liver and is a major source of energy needed to maintain liver viability during fasting. Beta-oxidation generates ketone bodies (acetoacetate, acetone and D-3-hydroxybutyrate), which serve as essential fuel sources for peripheral tissues, especially neurons, muscle and brain. Major defects in hepatic beta-oxidation cause microvesicular steatosis of the liver, intolerance to fasting and in some cases a myopathy. Although minor degrees of microvesicular steatosis can be seen in NASH, diseases characterized by microvesicular steatosis alone are invariably mitochondrial diseases and are reviewed extensively elsewhere (13).

The secretion of triglyceride into the circulation in the form of VLDL is the other mechanism of eliminating triglyceride from the hepatocyte. The synthesis and secretion of VLDL from the liver are complex and rate-limiting steps in the cycling of fat between the liver and peripheral stores (14). Through a process of combining triglyceride with apolipoprotein B100, phosphatidyl choline, cholesterol and cholesterol esters, functional VLDL is formed and secreted by exocytosis. Because of the multiple elements required for VLDL secretion, a wide variety of insults, genetic defects and deficiencies can impair its secretion. As a result, triglyceride accumulates, causing hepatic steatosis. This complexity explains why many seemingly unrelated causes of steatosis have this one clinical manifestation. Perhaps the most important cause in the context of treatment of NASH is the protein deficiency that can occur with fasting in an attempt to lose weight. Just as overt protein malnutrition (kwashiorkor) causes steatosis, fasting as a treatment of NASH can worsen the accumulation of fat in the liver $(15,16)$.

Finally, a link needs to be established between the accumulation of fat in the liver and the development of cell injury, inflammation and fibrosis. One hypothesis is that the fat within the steatotic liver serves as a readily available target for lipid peroxidation. Lipid peroxidation produces highly toxic intermediates that promote inflammation and directly compromise cellular integrity. Unfortunately, early reports of antioxidant regimens to treat NASH have not demonstrated significant benefit, with the exception of using vitamin $E$ in the pediatric population. An attractive alternative hypothesis suggests that overstimulation of hepatic Kupffer cells by gut-derived endotoxin may play an important role in mediating cell injury.

\section{CLINICAL FEATURES OF NASH}

Hepatic steatosis is typically asymptomatic, although some patients have right upper quadrant pain and a variety of nonspecific symptoms such as weakness, fatigue and malaise. The history must exclude alcohol abuse. What constitutes alcohol abuse in this context is probably more than $20 \mathrm{~g}$ daily for women and more than $60 \mathrm{~g}$ daily for men. A history should also be sought for environmental and occupational exposure to hydrocarbons such as working in the petrochemical industry (17). On examination, hepatomegaly is common but can be difficult to detect in the morbidly obese. Findings such as jaundice, spider angiomata, muscle wasting, ascites and palmar erythema point to the development of cirrhosis.

Most patients who come to medical attention for the evaluation of asymptomatic liver disease do so because of incidental laboratory abnormalities. Unfortunately, this selection bias confounds all clinical surveys that attempt to establish the sensitivity of laboratory testing for identifying 
patients with NASH. The experience gained from routine preoperative evaluation of patients undergoing surgical treatment of obesity has shown that progression of NASH to varying degrees of fibrosis and cirrhosis can occur with normal laboratory testing.

Nonetheless, the aminotransferases (alanine aminotransferase [ALT] and aspartate aminotransferase [AST]) provide the most useful information. NASH patients typically have serum aminotransferase levels that range from normal to four times the upper limit of normal. The presence of elevated aminotransferase levels cannot be used to distinguish benign steatosis from NASH because steatosis alone can cause elevations, even without evidence of overt cellular injury on liver biopsy.

The ratio of ALT to AST is often helpful in differentiating NASH from alcoholic steatohepatitis when the history is not convincing. Whereas patients with alcoholic hepatitis typically have serum AST levels that exceed ALT levels, patients with NASH almost invariably have an ALT level that is greater than the AST level in the absence of cirrhosis.

Other serum markers are less helpful. Gamma-glutamyltranspeptidase (GGT) elevations correlate with increased hepatic fat content, yet elevations of this enzyme are rarely isolated when substantial and rarely worth pursuing further when trivial. Serum triglyceride elevations are not seen uniformly in adults with NASH but are common in children with NASH.

\section{IMAGING}

The major abdominal imaging modalities, sonography, CT, magnetic resonance imaging (MRI) and radionuclide techniques, can contribute to the identification of focal or diffusely distributed hepatic steatosis, although none of these techniques differentiates benign hepatic steatosis from $\mathrm{NASH}$.

Ultrasonography is commonly used as a first step in the evaluation, but it can detect hepatic steatosis only when the degree of steatosis is substantial. Compared with the normal liver, the fatty liver is diffusely echogenic or 'bright'. The finding of a diffusely echogenic liver is not entirely specific to steatosis because cirrhosis can cause a similar appearance but with a subjectively coarser texture.

CT scanning typically reveals an abnormally low density liver compared with the spleen and paraspinal muscles even when intravenous contrast is employed. A low density liver is a relatively common finding, occurring in up to $10 \%$ of all abdominal CT scans. The low attenuation is typically diffuse, although in up to one-third of patients, the involvement is focal and appears as one or more low density lesions in an otherwise normal liver. Such focal fat can be found anywhere in the parenchyma or localized only to the periportal region. Focal sparing in a fatty liver can also occur; the resulting high density lesion can be confused with malignancy.

MRI can detect diffuse hepatic steatosis with a similar sensitivity to CT. The usefulness of MRI in further evaluating focal lesions suspected to be fat by CT is evolving, with phase shifting being employed to identify focal fat by its loss of intensity on T1-weighted images.

Radionuclide imaging using technetium-99m sulphur colloid (the 'liver-spleen scan') identifies focal areas of fat within the liver as focal defects, a finding that contributes nothing to distinguishing between benign and malignant etiologies. On the other hand, areas of focal sparing in the otherwise diffusely fatty liver take up tracer preferentially, and this can be helpful when evaluating such lesions. Hepatic ${ }^{133}$ xenon retention may offer the most sensitive measure of hepatic steatosis, but its clinical usefulness has not been extensively evaluated (18).

The incidental discovery of liver lesions caused by focal fat or focal sparing is a challenging and relatively common clinical problem. Even after employing more than one imaging modality, focal areas of fat in the liver can be difficult to distinguish from primary or metastatic malignancies. A few characteristics, such as a geometric appearance and the absence of a mass effect on adjacent structures, can suggest focal fat as the diagnosis. However, these are not always found, and fine-needle aspiration biopsy of problematic lesions may be required to exclude malignancy definitively, especially in patients with a history of prior malignancy. When the level of concern is lower, especially in children, repeated sonographic imaging within three to six months is a reasonable and more conservative approach. Focal sparing is often found in the caudate lobe because of aberrant portal venous drainage with isolation of the spared segments of the liver from intestinal venous blood. The location and geometric shape of focal sparing generally distinguish it from malignancy, but a fine-needle biopsy for histological confirmation may be necessary in unusual circumstances.

Fat within the liver causes several other imaging problems, especially with ultrasonography. Hemangiomas, which are characteristically echodense, may appear as relatively hypodense lesions when surrounded by a more echodense fatty liver. Dynamic CT scanning and tagged red cell radionuclide imaging are the best tests for establishing the identity of such lesions when the diagnosis is uncertain. Detection of intrahepatic biliary ductal dilation can be difficult in the fatty liver because the usual contrast between liver and the more echodense bile duct wall is lost.

\section{DIAGNOSIS}

The diagnosis of NASH is established only by liver biopsy $(6,19)$. The clinical setting, risk factors, laboratory data and imaging studies are only suggestive of the diagnosis, and all of these clinical parameters in aggregate remain relatively insensitive and nonspecific. Moreover, only the biopsy can distinguish benign steatosis from NASH. The threshold for obtaining a liver biopsy to evaluate patients suspected of having NASH should be low because early recognition can often provide the necessary incentive to achieve sustained weight loss in the obese. The role of a biopsy in patient management is also determined partly by the patient's desire to know if they have a potentially progressive form of liver disease. Finally, a diagnosis of NASH cannot be assumed on 
clinical grounds, and the biopsy sometimes identifies alternative causes of liver disease.

The biopsy findings in NASH are similar to those found in alcoholic hepatitis and include hepatocellular steatosis, lobular inflammation, Mallory bodies and pericellular zone 3 fibrosis (Figure 1). Despite that NASH can only be diagnosed by its histology, there remains controversy over which of these features are required for the diagnosis and which are not. This controversy will likely persist until the underlying causes of NASH are understood and the histological characteristics of each disorder that are lumped together in this entity called NASH are fully described individually. It was not so far back in the history of hepatology that debates around the significance of various features of an entity called chronic active hepatitis were debated. It can be argued that, with the separate identification of hepatitis C, hepatitis B and autoimmune hepatitis, the term chronic active hepatitis has lost its usefulness. So too may the definition of NASH evolve, but it cannot evolve without a beginning, and until pathogenesis has been defined, this remains the beginning.

\section{TREATMENT AND PROGNOSIS}

Weight loss can normalize elevated aminotransferase levels and reduce sonographically detectable hepatic fat in obese adults and obese children (20-22). The benefit of reducing or eliminating hepatic steatosis is twofold; symptoms such as right upper quadrant abdominal pain can be improved or eliminated, and the risk of developing progressive liver disease may be prevented. The amount of weight loss needed to normalize serum aminotransferase levels and reduce hepatic steatosis has not been rigorously established, but several studies have shown that only modest weight reduction may be needed (23). The means by which weight is lost is critically important. Gradual weight reduction achieved while providing adequate nutrients is crucial. Large caloric deficits can yield short term weight loss, but the flux of fat from peripheral stores to the liver in the setting of inadequate essential amino acids and other key nutrients can exacerbate the accumulation of hepatic fat.

Glycemic control in the type II diabetic patient may not provide the salutary effect that would be assumed based on the association between NASH and diabetes that has been reported in some studies. Weight reduction is much more important, and in the absence of significant weight reduction, liver enzyme levels in type II diabetic patients typically fail to improve, despite treatment with insulin to achieve better control of blood glucose (24). NASH is not a significant problem for patients with type I diabetes, although benign steatosis can develop with poor glycemic control. Symptomatic hepatomegaly caused by benign steatosis in type I diabetics responds to improved glycemic control.

The increasingly frequent identification of patients who are lean and do not have identifiable and correctable risk factors for NASH (1) has led to the search for alternative treatment strategies, with the aim of preventing progressive liver disease. Ursodeoxycholic acid may benefit a small subset of patients, and ongoing studies will hopefully provide
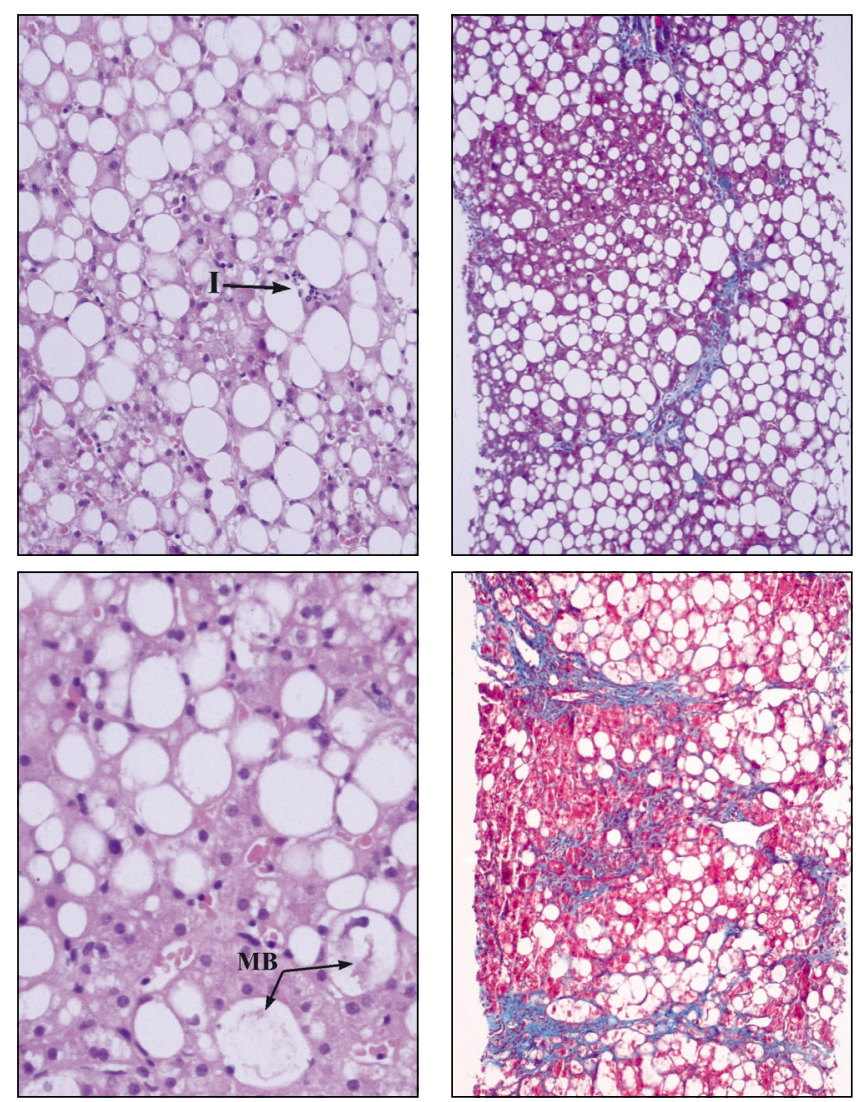

Figure 1) The biopsy findings of nonalcoholic steatohepatitis (NASH) are shown with hematoxylin and eosin-stained sections (Top left original magnification $\times 40$; Bottom left original magnification $\times 100)$ and Masson's trichrome-stained sections (Top right and Bottom right original magnification $\times 40)$. The steatosis found in NASH is predominantly macrovesicular (more than $2 \mu \mathrm{m}$ clear spaces that laterally displace hepatocyte nuclei). The distribution of fat can be panlobular or predominantly in zone 3 (perivenular). The inflammatory cells of NASH are a mix of neutrophils and mononuclear cells and are found throughout the lobule (I). Although focal neutrophilic infiltrates can be found in areas of focal necrosis, neutrophils are not usually the predominant inflammatory cell type. The presence of Mallory bodies $(\mathrm{MB})$ and glycogen nuclei are more variable findings in NASH. MB appear as eosinophilic, stranded structures in the hepatocyte cytoplasm and are identified most easily in ballooned hepatocytes. The MB of NASH are often small compared with the $\mathrm{MB}$ found in alcoholic hepatitis, and the presence of large MB should raise the suspicion of surreptitious alcohol abuse. Glycogen nuclei are seen more frequently in NASH than alcoholic liver disease. They are a nonspecific finding common in diabetes, Wilson's disease and other disorders. The pattern of early fibrosis (C and D, blue) in NASH is similar to alcoholic liver disease, with collagen around the central vein and in the zone 3 perisinusoidal spaces producing a chicken wire appearance

clues as to who might be most likely to respond (25). The underlying reason why this agent may be helpful remains unknown. Vitamin E supplementation was found to be effective in reducing aminotransferase levels and sonographically detectable fat in children (26), although children may be a more homogeneous population with respect to pathogenesis than adults. Choline deficiency was a major cause of hepatic steatosis during prolonged administration of parenteral nutrition before the solutions were routinely supplemented with additional choline. Pantothenic acid deficiency has 
also been proposed as a cause of hepatic steatosis. Pantothenic acid is necessary for the synthesis of coenzyme $A$, and coenzyme A is essential for hepatic fat metabolism. In one unblinded study, pantothenic acid supplementation was thought to cause histological improvement in hepatic steatosis. Well designed clinical studies are needed to define who might benefit from such therapeutic interventions. Because of the complexity of fat secretion from the liver and the subsequent secondary stress that leads to inflammation and fibrosis, there are likely many different causes of NASH that will each require its own specific treatment.

As a last option, liver transplantation can be considered for selected patients who progress to decompensated cirrhosis. About $1 \%$ of transplantations at major centres are performed on patients with known, pre-existing NASH. This figure may underestimate the true prevalence of end-stage

\section{REFERENCES}

1. Bacon BR, Farahvash MJ, Janney CG, Neuschwander-Tetri BA. Nonalcoholic steatohepatitis: an expanded clinical entity. Gastroenterology 1994;107:1103-9.

2. Galambos JT, Wills CE. Relationship between 505 paired liver tests and biopsies in 242 obese patients. Gastroenterology 1978;74:1191-5.

3. Baldridge AD, Perez-Atayde AR, Graeme-Cook F, Higgins L, Lavine JE. Idiopathic steatohepatitis in childhood: a multicenter retrospective study. J Pediatr 1995;127:700-4.

4. Itoh S, Yougel T, Kawagoe K. Comparison between nonalcoholic steatohepatitis and alcoholic hepatitis. Am J Gastro 1987;82:650-4

5. Lee RG. Nonalcoholic steatohepatitis: a study of 49 patients. Hum Pathol 1989;20:594-8.

6. Ludwig J, Viggiano TR, McGill DB, Ott BJ. Nonalcoholic steatohepatitis. Mayo Clin Proc 1980;55:434-8.

7. Wanless IR, Lentz JS. Fatty liver hepatitis (steatohepatitis) and obesity: an autopsy study with analysis of risk factors. Hepatology 1990;12:1106-10.

8. Ikai E, Ishizaki M, Suzuki Y, Ishida M, Noborizaka Y, Yamada Y. Association between hepatic steatosis, insulin resistance and hyperinsulinaemia as related to hypertension in alcohol consumers and obese people. J Hum Hyperten 1995;9:101-5.

9. Batman PA, Scheuer PJ. Diabetic hepatitis preceding the onset of glucose intolerance. Histopathology 1985;9:237-43.

10. Powell EE, Cooksley WG, Hanson R, Searle J, Halliday JW, Powell LW. The natural history of nonalcoholic steatohepatitis: a follow-up study of forty-two patients for up to 21 years. Hepatology 1990;11:74-80.

11. Powell EE, Searle J, Mortimer R. Steatohepatitis associated with limb lipodystrophy. Gastroenterology 1989;97:1022-4.

12. el-Hassan AY, Ibrahim EM, al-Mulhim FA, Nabhan AA, Chammas MY. Fatty infiltration of the liver: analysis of prevalence, radiological and clinical features and influence on patient management. Br J Radiol 1992;65:774-8.

13. Fromenty B, Berson A, Pessayre D. Microvesicular steatosis and steatohepatitis: role of mitochondrial dysfunction and lipid peroxidation. J Hepatol 1997;26(Suppl 1):13-22. liver disease caused by NASH because the histological findings of NASH can resolve after the development of cirrhosis, and patients subsequently present with bland cryptogenic cirrhosis. Provocative evidence linking abnormalities of hepatic fat metabolism to the development of cirrhosis is the disproportionately high prevalence of mutations in the apolipoprotein B100 lipoprotein gene in patients with cryptogenic cirrhosis. After transplantation, NASH may recur in about one-third of patients, suggesting that persistent peripheral metabolic abnormalities or infectious causes could be contributing factors.

In summary, gradual and sustained weight reduction is the mainstay of therapy for the overweight. Other therapies have not been as beneficial, and until more is known about the pathogenesis of NASH, effective alternative treatments, especially for the nonoverweight, may be lacking.

14. Gruffat D, Durand D, Graulet B, Bauchart D. Regulation of VLDL synthesis and secretion in the liver. Reprod Nutr Dev 1996;36:375-89.

15. Capron JP, Delamarre J, Dupas JL, et al. Fasting in obesity: another cause of liver injury with alcoholic hyaline? Dig Dis Sci 1982;27:265-8.

16. Rozental P, Biava C, Spencer H, et al. Liver morphology and function tests in obesity and during starvation. Am J Dig Dis 1967;12:198-208

17. Cotrim HP, Freitas LA, Parana R, et al. Non-alcoholic steatohepatitis (NASH) and environmental toxins: A liver disease in workers from an industrial area. Hepatology 1986;24:337.

18. Yeh SH, Wu LC, Wang SJ, et al. Xenon-133 hepatic retention ratio: a useful index for fatty liver quantification. J Nucl Med 1989;30:1708-12.

19. Lee RG. Nonalcoholic steatohepatitis: tightening the morphological screws on a hepatic rambler. Hepatology 1995;21:1742-3.

20. Vajro P, Fontanella A, Perna C, Orso G, Tedesco M, De Vincenzo A. Persistent hyperaminotransferasemia resolving after weight reduction in obese children. J Pediatr 1994;125:239-41.

21. Drenick EJ, Simmons F, Murphy JF. Effect on hepatic morphology of treatment of obesity by fasting, reducing diets and small bowel bypass. N Engl J Med 1970;282:829-34.

22. Fiatarone JR, Coverdale SA, Batey RG, Farrell GC. Non-alcoholic steatohepatitis: impaired antipyrine metabolism and hypertriglyceridaemia may be clues to its pathogenesis. J Gastroenterol Hepatol 1991;6:585-90.

23. Schaffner F, Thaler H. Nonalcoholic fatty liver disease. Prog Liver Dis 1986;8:283-98.

24. Seeff LB, Zimmerman HI. Relationship between hepatic and pancreatic disease. In: Popper H, Schaffner F, eds. Progress in Liver Diseases, vol 5. New York: Grune and Stratton, 1976:590.

25. Laurin J, Lindor KD, Crippin JS, et al. Ursodeoxycholic acid or clofibrate in the treatment of non-alcohol-induced steatohepatitis: a pilot study. Hepatology 1996;23:1464-7.

26. Lavine JE. Treatment of obesity-induced steatohepatitis with vitamin E. Gastroenterology 1998;114:A1284-5. (Abst) 


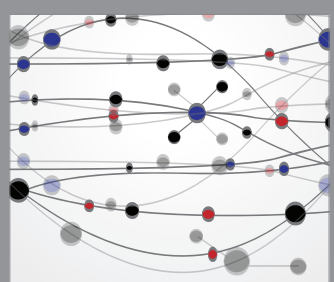

The Scientific World Journal
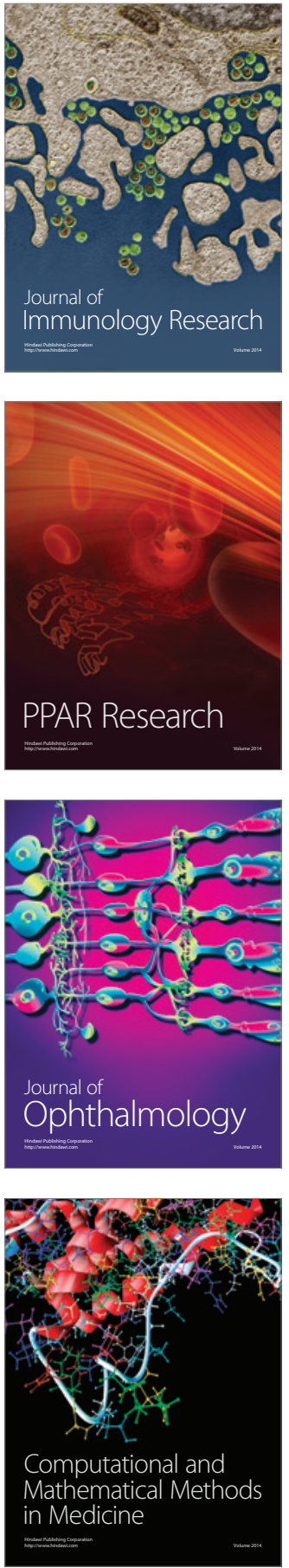

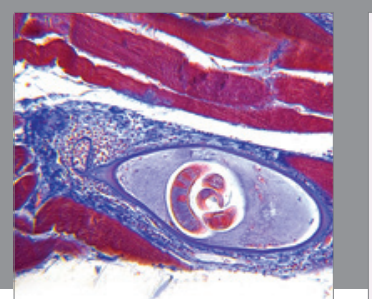

Gastroenterology Research and Practice

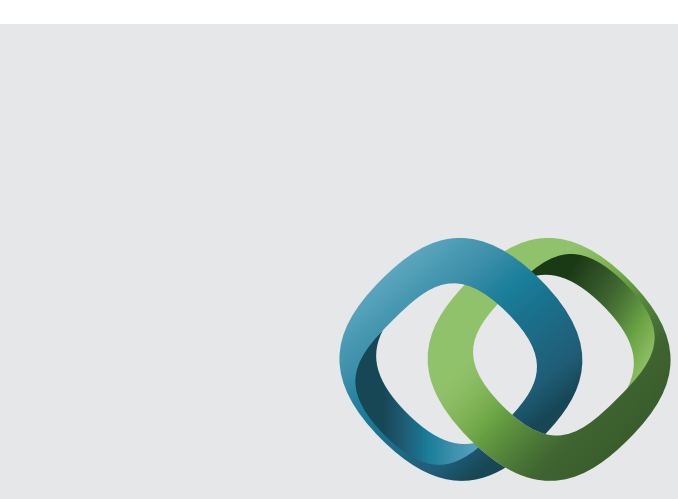

\section{Hindawi}

Submit your manuscripts at

http://www.hindawi.com
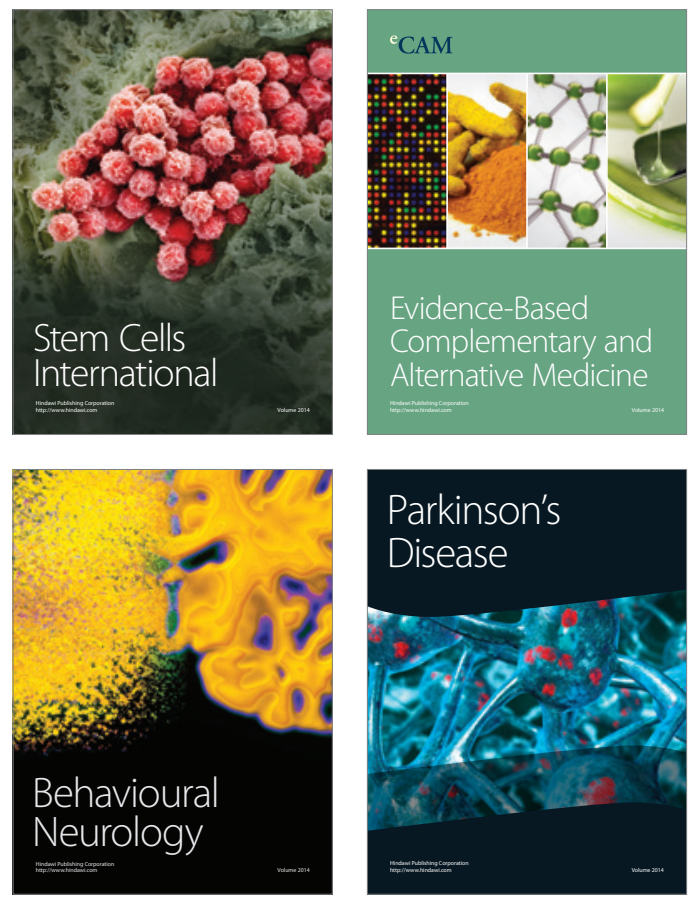
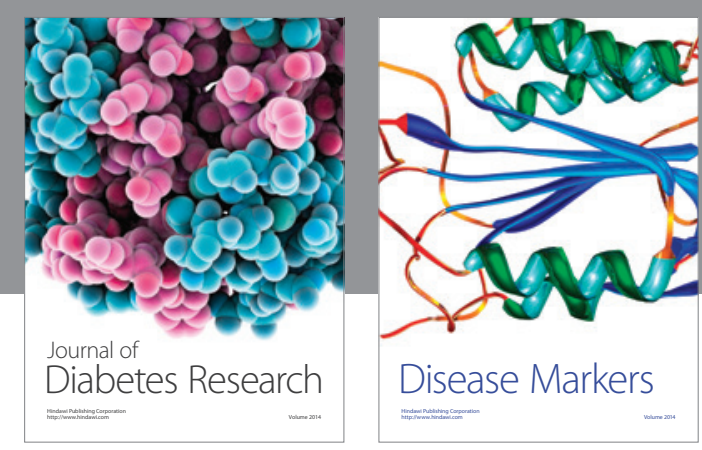

Disease Markers
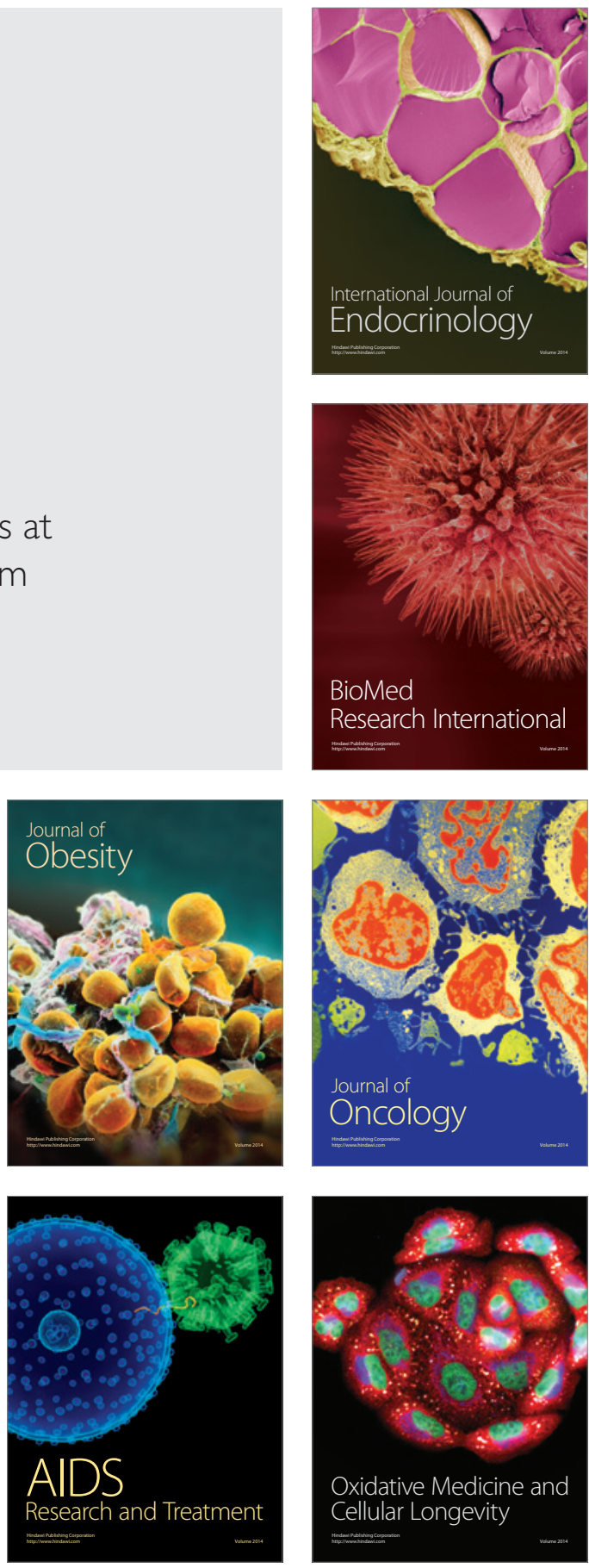